\title{
On how high performers keep cool brains in situations of cognitive overload
}

\author{
Susanne M. JAeggi And Martin BuschKuehl \\ University of Bern, Bern, Switzerland \\ Alex Etienne \\ University of Neuchâtel, Neuchâtel, Switzerland \\ Christoph Ozdoba \\ University Hospital of Bern, Bern, Switzerland \\ Walter J. Perrig \\ University of Bern, Bern, Switzerland \\ AND \\ Arto C. NiRKKo \\ University Hospital of Bern, Bern, Switzerland
}

\begin{abstract}
What happens in the brain when we reach or exceed our capacity limits? Are there individual differences for performance at capacity limits? We used functional magnetic resonance imaging (fMRI) to investigate the impact of increases in processing demand on selected cortical areas when participants performed a parametrically varied and challenging dual task. Low-performing participants respond with large and load-dependent activation increases in many cortical areas when exposed to excessive task requirements, accompanied by decreasing performance. It seems that these participants recruit additional attentional and strategy-related resources with increasing difficulty, which are either not relevant or even detrimental to performance. In contrast, the brains of the high-performing participants "keep cool" in terms of activation changes, despite continuous correct performance, reflecting different and more efficient processing. These findings shed light on the differential implications of performance on activation patterns and underline the importance of the interindividual-differences approach in neuroimaging research.
\end{abstract}

Increasing demands in cognitive tasks are often correlated with increasing stress for the performer. The risk of a breakdown in performance at the edge of mental overload creates an emergency state in which calmness, combined with fully functional thinking and focused attention, is the key determinant for making the most of the situation. There are many settings in everyday life in which good performers can turn potential harm into accomplishment. In many professional domains, society is concerned to select and train such individuals for demanding activities. Therefore, learning about what happens in the brain when we reach and exceed our capacity limits while performing complex tasks and learning more about individual differences in performing these tasks not only is of relevance for basic research, but also has practical implicationsfor example, when staff designated for work in highly demanding situations is selected.

In this study, we used a brain-imaging approach to examine individual differences in cognitive performance at the limits of working memory (WM) capacity and beyond. $\mathrm{WM}$, in this context, is seen as "the set of mental processes holding limited information in a temporarily accessible state in service of cognition" (Cowan et al., 2005, p. 42) and seems to predict a wide variety of higher order cognitive tasks (Daneman \& Carpenter, 1980; Kyllonen \& Christal, 1990). Capacity limits in WM are expressed as decreasing performance in response to increasing demands. The nature of capacity limits has been discussed extensively in the literature (e.g., Cowan, 2001, 2005), but there still is no consensus about the exact definition of the limits and how best to characterize them. Also, little is known about the underlying neural processes as the capacity limits of WM are reached and, especially, exceeded. Numerous functional neuroimaging studies have consistently reported load-dependent activation changes in prefrontal areas (e.g., Braver et al., 1997; Carlson et al., 1998; Linden et al., 2003; Nystrom et al., 2000; Owen, McMillan, Laird, \& Bullmore, 2005; Schumacher et al., 1996) and 
also in parietal cortices (e.g., Linden et al., 2003; Todd \& Marois, 2004; Xu \& Chun, 2006). Although WM load has been parametrically manipulated in these studies, it has been restricted to relatively easy levels and usually has not explicitly challenged or exceeded the capacity limits of the participants, which was the focus of our interest in the present study. In a review, Kane and Engle (2002) have argued that the prefrontal cortices (PFCs) are critical for effective WM capacity and have suggested that individual differences in WM capacity might also be mirrored by individual differences in prefrontal areas, especially in the dorsolateral PFC (DLPFC). In the neuroimaging literature, some authors have indeed observed prefrontal activation changes in response to task load - that is, a decrease in prefrontal activation at high levels of load, which has been interpreted as a breakdown in neural networks (Goldberg et al., 1998), as the neurophysiological response to the capacity-constrained WM system (Callicott et al., 1999; Perlstein, Carter, Noll, \& Cohen, 2001) - or in dysfunctional WM-related processing in schizophrenic patients (Jansma, Ramsey, van der Wee, \& Kahn, 2004; Perlstein et al., 2001). In contrast, other researchers have reported increasing bilateral activation in dorsolateral prefrontal areas with supracapacity memory loads (Rypma \& D'Esposito, 1999), providing evidence for what they view as the involvement of executive processes. Integrating these contradictory results, several researchers have proposed that increases in memory load should be reflected by an inverted U-shaped activation curve or capacity-constrained response from lowest to highest load (Callicott et al., 1999), especially in dual-task situations (D'Esposito, 2001), where processing limitations become obvious quite soon and also reflect common demands of everyday life. Still, our own work in which dual tasks were parametrically varied yielded no evidence of a decrease in prefrontal activation in such conditions - that is, in the face of excessive memory demands (Jaeggi et al., 2003). Rather, even with demands for which performance was at chance level, activation still increased in the DLPFC and other areas relating to the WM network, such as superior parietal and premotor areas. This finding was interpreted in terms of motivational factors - that is, that the participants tried to succeed even though the task was very difficult, which is in line with an effortful attention hypothesis (Frith \& Dolan, 1996; Ingvar, 1994). A decrease of activation is, therefore, expected only "as subjects become overwhelmed and subsequently disengage from the task" (Callicott et al., 1999, p. 25).

On the basis of these results, we hypothesized that the U-shaped activation curve in prefrontal cortices, as proposed in the literature, applies only to specific conditions also when individual differences are taken into consideration: A decrease in cortical activation in the dual task at high levels of load will occur only if participants exceed their individual capacity limits and, more important, if they give up trying to succeed. We assessed these mediating factors with behavioral recordings (accuracy and reaction time [RT]) and with a qualitative posttest inventory. We predicted a differential activation pattern for high- and low-performing participants. Low-performing participants were expected to show a decrease of activation in the most difficult dual-task condition (when they reach their capacity limits and disengage from the task). From high-performing participants, who might still be able to perform the task adequately, an ongoing increase of activation was expected. Besides the prefrontal areas, other, more posterior areas are also assumed to play a role in tasks performed at capacity limits (e.g., Linden et al., 2003; Todd \& Marois, 2004; Xu \& Chun, 2006). Thus, various other regions of interest were evaluated in order to include a whole task-related network and in order to investigate whether differential activation patterns between participants would also be observed in other, nonprefrontal cortical regions.

In order to test the predictions laid out in the previous paragraph, we used a sequential and parametric $n$-back task at four levels of difficulty, performed as a dual task with simultaneous presentation of verbal auditory and visuospatial material, which had to be processed independently (see Figure 1).

\section{METHOD}

\section{Participants}

Fifteen young adults ( 9 women and 6 men; age range, 21-29 years; mean age, 25 years, 4 mos.) participated in this study. All were assessed as right-handed (Oldfield, 1971), with no seeing, hearing, or neurological disorders and with normal structural MRI scans. All the participants were given identical time to practice the task before scanning: All the tasks were practiced in the experimental laboratory beforehand in a manner similar to that for the fMRI session. However, the sequence of the different $n$-back tasks varied, being randomly assigned by the program in order to prevent position effects. On the basis of their performance levels in the most difficult task (3-back dual; accuracy) in the fMRI-session, two groups of participants were differentiated on the basis of a median split, consisting of 8 low-performing participants ( 5 men and 3 women) and 7 high-performing participants (1 man and 6 women).

\section{Stimulus Materials and Procedure}

A sequential $n$-back paradigm (Braver et al., 1997; Jonides et al., 1997; Kirchner, 1958) was used at four levels of difficulty, administered as a dual task. Visuospatial and auditory-verbal material was presented simultaneously; however, the participants had to process both modalities independently (see Figure 1A). For the 0 -back condition, serving as baseline condition with minimal memory demands, the participant was requested to respond to a prespecified target (i.e., "Q" for the auditory condition and/or "upper left corner" for the visuospatial condition), whereas for the other conditions, a response was required whenever the current stimulus matched the stimulus 1 , 2 , or 3 positions back in the sequence. The task load was always the same for both modalities. The visuospatial stimuli consisted of blue squares, appearing in eight different loci on the computer screen. The auditory material consisted of eight German consonants (c, g, $\mathrm{h}, \mathrm{k}, \mathrm{p}, \mathrm{q}, \mathrm{t}$, and w) spoken in a female voice and selected on the basis of their distinctiveness. We used a blocked periodic design in which the stimuli were presented to the participants during epochs of $30 \mathrm{sec}$ ( 0 -back) or $60 \mathrm{sec}$ (1-back to 3-back), always preceded by instruction templates for $15 \mathrm{sec}$ (see Figure 1B). The positions of the 1- to 3-back tasks were determined randomly, each appearing twice and always followed and preceded by the 0-back task, which served as the baseline task. All the conditions were matched for number of targets presented (33\%) and consisted of 70 trials in the 0 -back task and of 40 trials in each 1-, 2-, and 3-back task. One trial consisted of 


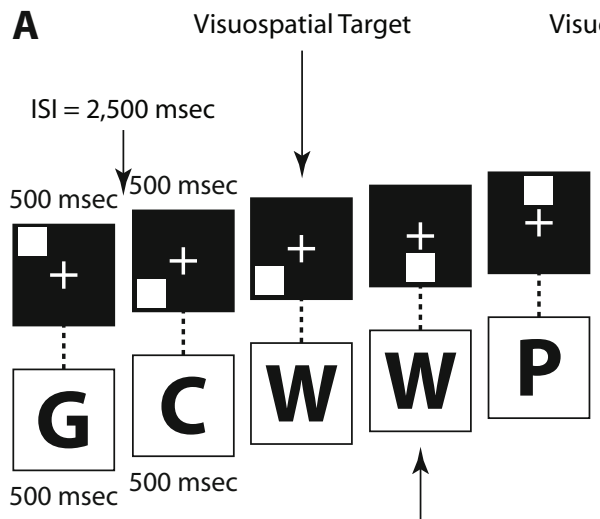

Auditory Target
Visuospatial Target
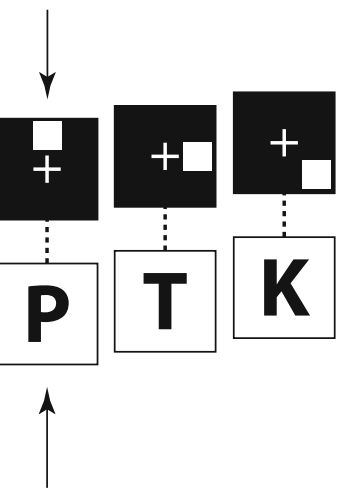

Auditory Target
Visuospatial Target

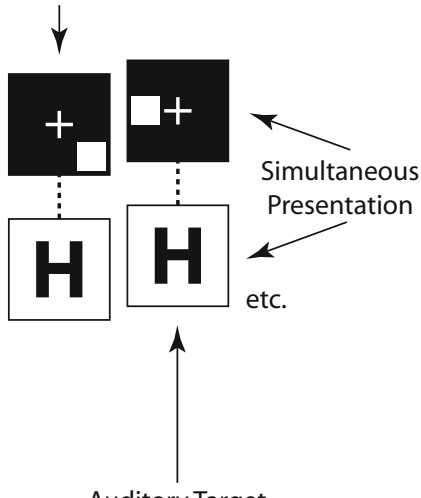

Auditory Target

\section{1-Back Dual Task}
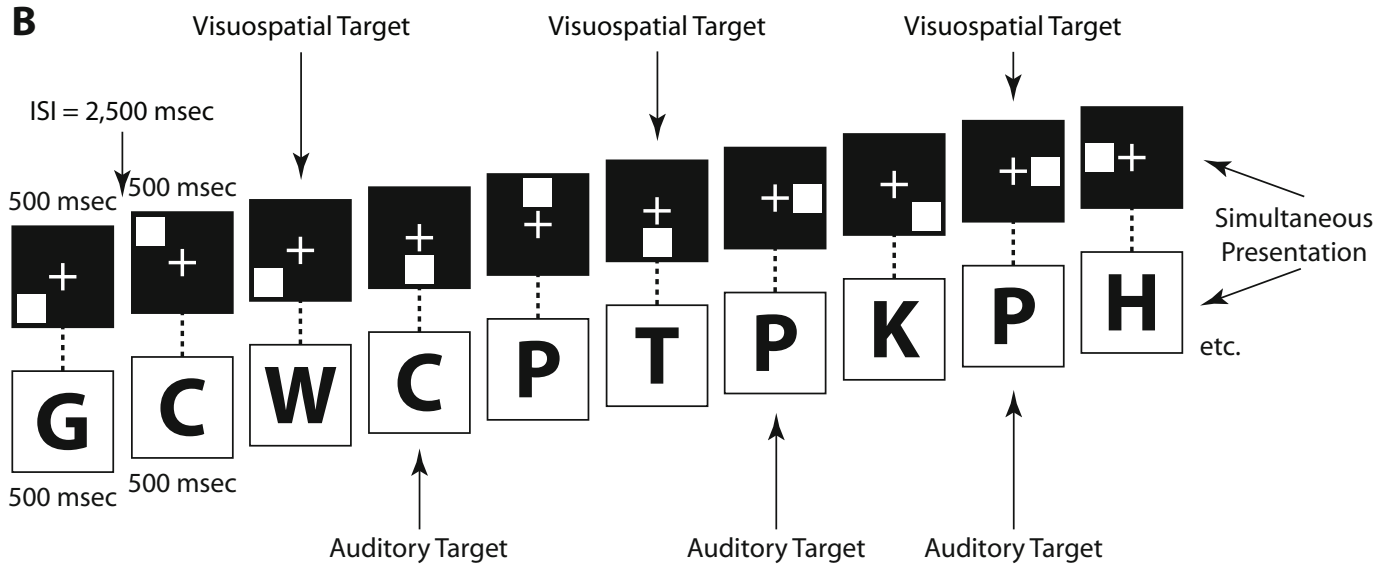

2-Back Dual Task

$60 \mathrm{sec}$

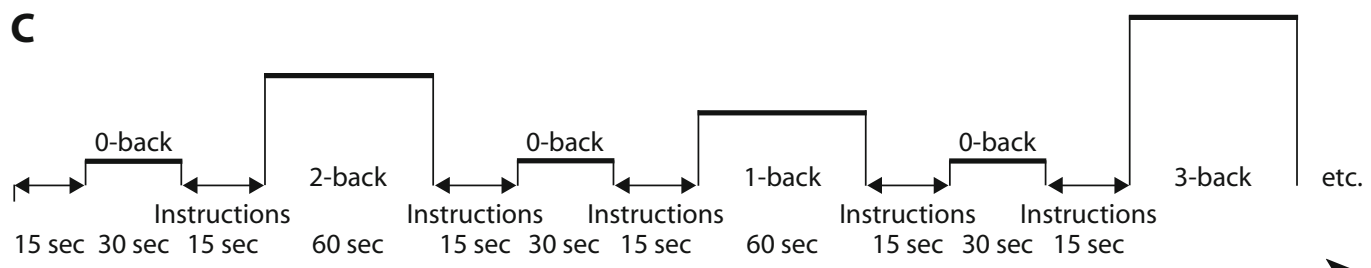

$13 \min$

Figure 1. The $n$-back task. Stimuli were presented simultaneously for both modalities (visual and auditory). The participants had to simultaneously pay attention to both modalities, and responses were required independently for each modality whenever the current stimulus matched the stimulus one, two, or three positions back in the sequence (depending on the load level); no response was requested to nontargets. For the 0-back condition, serving as baseline condition with minimal memory demands, the participant had to respond to a prespecified target (i.e., "Q" for the auditory condition and "upper left corner" for the visuospatial condition). (A) Exemplary 1-back condition. (B) Exemplary 2-back condition (ISI, interstimulus interval, a fixation cross). (C) Task procedure and alternations (block design) for the $n$-back task.

one stimulus ( $500 \mathrm{msec})$ and one interstimulus interval $(2,500 \mathrm{msec})$ which was a white fixation cross on a black screen. Targets and nontargets were determined pseudorandomly - that is, by varying the position of the targets in each sequence randomly while holding the number of interfering distractors (e.g., 2-back targets in a 3-back condition) constant. The targets could occur either in only one or in both modalities at the same time. The participants responded to the targets by pressing a pneumatic squeeze ball (left hand for visual stimuli; right hand for auditory-verbal stimuli); no responses were required for nontargets.

With a qualitative posttest inventory, we assessed the used strategies for each task load.

\section{fMRI Method}

After shimming, a whole-brain high-resolution fMRI was performed with a blood oxygenation level dependent (BOLD) echo- 
planar imaging $($ EPI) sequence (matrix, $128 \times 128 \times 48$ slices $\times$ 108 measurements, resulting in a resolution of $1.5 \times 1.5 \times 3 \mathrm{~mm} \times$ TR 7,500 msec; TE, $60 \mathrm{msec}$ ) on a standard clinical 1.5-T wholebody MRI scanner (Magnetom Vision, Siemens Medical Systems, Erlangen, Germany). Slice angulation was parallel to a line connecting the base of the genu corporis callosi and the confluens sinuum, resulting in an angle of about $20^{\circ}-30^{\circ}$ with respect to the bicommissural (AC-PC) line. In addition, standardized sets of high-resolution structural images were acquired for later coregistration. The experiment was controlled by a Microsoft-Windows-based personal computer using E-Prime (Psychology Software Tools, Pittsburgh, PA). Stimuli were visually presented to the participants as they were lying in the scanner via a prismatic mirror and were aurally presented via headphones.

\section{Data Analysis}

Behavioral data. For behavioral data, RT and accuracy (i.e., $P_{\mathrm{r}}$; hits minus false alarms, according to Snodgrass \& Corwin, 1988) were analyzed. As was described above, groups of high- and low-performing participants were differentiated post hoc by a median split based on their accuracy levels $\left(P_{\mathrm{r}}\right)$ in the most difficult task (3-back) in the fMRI-session. A two-way repeated measures ANOVA was calculated for both behavioral measures (accuracy and RT) with load (0-back to 3-back) as within-subjects factor and performance group (high and low performer) as between-subjects factor, and post hoc $t$ tests (two-tailed) were applied in case of a significant interaction and were Bonferroni corrected for multiple comparisons. Furthermore, test-retest reliability was estimated for $P_{\mathrm{r}}$ with product-moment correlations (Pearson's) between the practice and the fMRI sessions for each level of load.

fMRI data. For fMRI evaluation, $z$ score maps were generated after spatial filtering and were projected onto original EPI images, using self-developed software (Jaeggi et al., 2003; Nirkko, 2000). To compare the amount of activation in a given area across experimental conditions, a priori volumes of interest (VOIs) were defined using the individual anatomical parcellation method according to Rademacher, Galaburda, Kennedy, Filipek, and Caviness (1992). The same VOIs were defined for each participant, thus accounting for individual differences in brain geometry. The VOIs were manually delineated on each functional slice, also with self-developed software (Jaeggi et al., 2003; Nirkko et al., 2001). Thirty VOIs in each hemisphere were defined in total, taking into account the meta-analytic findings concerning the $n$-back task (Owen et al., 2005) and WM in general (Wager \& Smith, 2003). The cortex was completely covered with these VOIs, which were delineated on each individual participant's anatomy, resulting in two major advantages.

Table 1

Mean Size (Number of Pixels and Volume) and Standard Deviation for Each Defined Volume of Interest (VOI)

\begin{tabular}{|c|c|c|c|c|c|c|c|c|c|c|}
\hline \multirow[b]{3}{*}{ Brain Area } & \multirow[b]{3}{*}{ Parcellation Units (VOIs) } & \multirow[b]{3}{*}{ Abbr. } & \multicolumn{4}{|c|}{ Left Hemisphere } & \multicolumn{4}{|c|}{ Right Hemisphere } \\
\hline & & & \multicolumn{2}{|c|}{$\begin{array}{l}\text { Number of } \\
\text { Pixels }\end{array}$} & \multicolumn{2}{|c|}{$\begin{array}{l}\text { Volume } \\
\left(\mathrm{mm}^{3}\right)\end{array}$} & \multicolumn{2}{|c|}{$\begin{array}{l}\text { Number of } \\
\text { Pixels }\end{array}$} & \multicolumn{2}{|c|}{$\begin{array}{l}\text { Volume } \\
\left(\mathrm{mm}^{3}\right)\end{array}$} \\
\hline & & & $M$ & $S D$ & $M$ & $S D$ & $M$ & $S D$ & $M$ & $S D$ \\
\hline \multicolumn{11}{|l|}{ Lateral cerebral surface } \\
\hline \multirow[t]{8}{*}{ Frontal lobe } & Central sulcus & ce & 2,457 & 430 & 16,583 & 2,905 & 2,474 & 545 & 16,700 & 3,680 \\
\hline & Superior frontal gyrus & SFG & 2,224 & 447 & 15,014 & 3,014 & 2,298 & 363 & 15,512 & 2,449 \\
\hline & Middle frontal gyrus & DLPFC & 3,657 & 643 & 24,684 & 4,342 & 3,589 & 601 & 24,223 & 4,058 \\
\hline & Frontal eye fields & FEF & 330 & 101 & 2,229 & 683 & 324 & 147 & 2,188 & 990 \\
\hline & Frontal pole & FP & 792 & 221 & 5,348 & 1,494 & 745 & 248 & 5,027 & 1,671 \\
\hline & Inferior frontal gyri & IFG & 1,615 & 238 & 10,899 & 1,604 & 1,847 & 479 & 12,465 & 3,233 \\
\hline & Precentral gyrus & PRG & 2,073 & 462 & 13,990 & 3,116 & 2,061 & 488 & 13,912 & 3,294 \\
\hline & Superior frontal sulcus & sf & 2,529 & 623 & 17,071 & 4,207 & 2,563 & 634 & 17,298 & 4,279 \\
\hline \multirow[t]{3}{*}{ Parietal lobe } & Inferior parietal lobule & INFPL & 1,693 & 530 & 11,430 & 3,579 & 1,613 & 405 & 10,890 & 2,736 \\
\hline & Superior parietal lobule & SPL & 3,186 & 521 & 21,502 & 3,518 & 2,970 & 508 & 20,048 & 3,426 \\
\hline & Postcentral gyrus & POG & 2,102 & 609 & 14,190 & 4,113 & 2,173 & 568 & 14,666 & 3,835 \\
\hline \multirow[t]{3}{*}{ Temporal lobe } & Inferior temporal sulcus & it & 1,706 & 543 & 11,516 & 3,664 & 1,882 & 428 & 12,707 & 2,890 \\
\hline & Superior temporal sulcus & st & 2,857 & 556 & 19,285 & 3,756 & 3,001 & 512 & 20,254 & 3,456 \\
\hline & Temporal pole & $\mathrm{TP}$ & 408 & 150 & 2,753 & 1,012 & 390 & 112 & 2,630 & 759 \\
\hline \multirow[t]{5}{*}{ Occipital lobe } & Intracalcarine cortex & calc & 1,651 & 324 & 11,142 & 2,185 & 1,777 & 325 & 11,992 & 2,197 \\
\hline & $\begin{array}{l}\text { Lateral occipital cortex, } \\
\text { inferior division }\end{array}$ & $\mathrm{OLi}$ & 2,189 & 395 & 14,777 & 2,667 & 2,299 & 464 & 15,518 & 3,130 \\
\hline & Lateral occipital cortex, & & & & & & & & & \\
\hline & superior division & OLs & 1,711 & 354 & 11,551 & 2,389 & 1,735 & 411 & 11,712 & 2,771 \\
\hline & Occipital pole & $\mathrm{OP}$ & 1,110 & 345 & 7,493 & 2,328 & 1,180 & 478 & 7,965 & 3,228 \\
\hline \multicolumn{11}{|l|}{ Medial cerebral surface } \\
\hline \multirow[t]{4}{*}{ Frontal lobe } & Anterior cingulate gyrus & $\mathrm{CGa}$ & 1,605 & 406 & 10,832 & 2,742 & 1,715 & 414 & 11,579 & 2,791 \\
\hline & Paracingulate gyrus & PAC & 1,774 & 251 & 11,975 & 1,692 & 1,679 & 239 & 11,333 & 1,611 \\
\hline & Supplementary & & & & & & & & & \\
\hline & motor cortex & SMC & 645 & 134 & 4,357 & 905 & 607 & 118 & 4,099 & 797 \\
\hline \multirow[t]{2}{*}{ Parietal lobe } & Posterior cingulate gyrus & CGp & 852 & 266 & 5,752 & 1,794 & 828 & 254 & 5,591 & 1,717 \\
\hline & Precuneus & $\mathrm{PCN}$ & 1,948 & 441 & 13,150 & 2,975 & 2,067 & 530 & 13,954 & 3,578 \\
\hline \multirow[t]{3}{*}{ Intrasylvian cerebral surface } & Heschl sulcus & he & 319 & 187 & 2,150 & 1,261 & 325 & 126 & 2,196 & 850 \\
\hline & Insular cortex & INS & 2,495 & 447 & 16,838 & 3,018 & 2,436 & 483 & 16,444 & 3,258 \\
\hline & Sylvian fissure & sy & 939 & 271 & 6,337 & 1,832 & 834 & 213 & 5,627 & 1,439 \\
\hline \multirow[t]{3}{*}{ Subcortical structures } & Caudate nucleus & cau & 282 & 60 & 1,903 & 406 & 283 & 72 & 1,908 & 488 \\
\hline & Putamen & $\mathrm{pu}$ & 479 & 98 & 3,234 & 662 & 493 & 106 & 3,327 & 713 \\
\hline & Thalamus & thal & 647 & 124 & 4,365 & 836 & 652 & 139 & 4,400 & 935 \\
\hline Cerebellum & Cerebellar hemisphere & $\mathrm{CH}$ & 5,353 & 871 & 36,135 & 5,878 & 5,416 & 709 & 36,558 & 4,787 \\
\hline
\end{tabular}

Note $-N=15$. 
First, a reliable interparticipant match between structurally corresponding regions could be achieved without the interparticipant localization error inherent to voxel-based group evaluation following automatic normalization procedures. This also results in better statistical reliability, since a participant's VOIs are not as much contaminated by neighboring, overlapping, or otherwise mislocalized regions as is the case with standard normalization methods. Second, the massively reduced amount of independent units to be statistically assessed and Bonferroni corrected (60 VOIs instead of a few 100,000 pixels or an intermediate amount of resolution elements ["resells"]) results in a greatly improved sensitivity while still covering the whole brain.

The VOI sizes are described in Table 1 and depicted in Figure 2. Dorsolaterally, we defined eight frontal VOIs: the superior frontal gyrus (SFG), comprising the superior portions of Brodman's areas (BAs) 6, 8, and 9; the middle frontal gyrus (DLPFC/F2; BAs 6, 8, 9 , and 46), both with the exception of the posterior-most part (the frontal eye fields; FEFs) in order to exclude activation related predominantly to eye movements; the inferior frontal gyri (IFGs) consisting of the orbital, pars triangularis, and pars opercularis (BAs 47, 45 , and 44); the premotor cortex (PRG) adjacent to the precentral sulcus at the level of the "hand notch" (BA 6); the frontal pole (FP; BA 10); and the cortical areas adjacent to their defining sulci-that is, the central sulcus (ce; BAs 4, 1, 2, and 3) and the superior frontal sulcus (sf). Furthermore, we defined three parietal VOIs (i.e., the region adjacent to the postcentral gyrus [BAs 5 and 7], the inferior parietal lobule [INFPL], comprising inferior portions of the posterior supramarginal and the angular gyrus [BAs 7, 40, and 39], and the superior parietal lobule [SPL; BAs 5 and 7]), three temporal VOIs (the temporal pole [TP; BA 38] and the areas including and adjacent to the inferior temporal [it] and superior temporal [st] sulci [BAs 22, 21, and 37]), and finally, four occipital VOIs (the intracalcarine cortex [calc; BA 17, 18], the lateral superior and inferior occipital cortex [OLs, OLi; BAs 18 and 19], and the occipital pole [OP; BA 17]). Medially, we defined three frontal VOIs
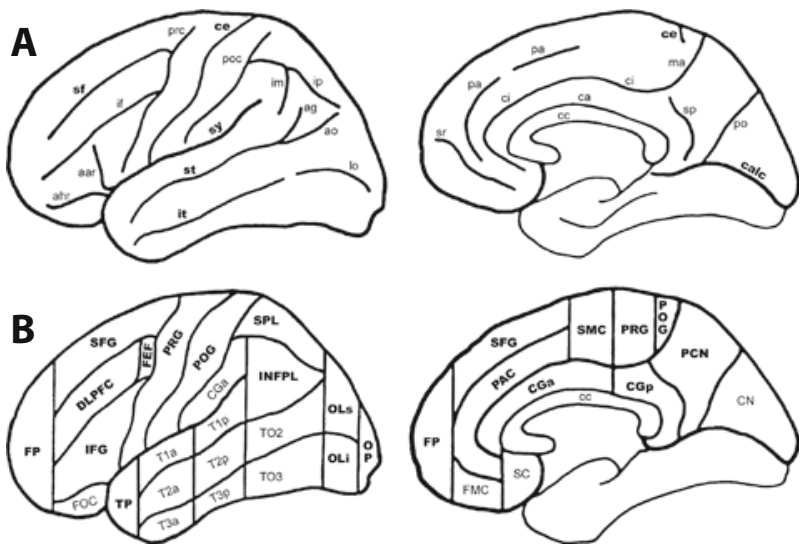

Figure 2. Schematic diagram of the individually defined volumes of interest (VOIs) based on the parcellation method by Rademacher (1992). Some VOIs were further refined and adapted by us; that is, some parcellation units were joined into larger VOIs. Thirty VOIs were defined in each hemisphere, which are indicated with bold font. (A) Idealized topography of limiting sulci (lowercase). Note that the defined sulci are usually taken as areas including and adjacent to the representative sulci-that is, the intracalcarine cortex (calc), inferior and superior temporal sulci (it and st), Heschl's gyri (he), Sylvian fissure (sy), superior frontal gyrus (sf), and central sulcus (ce). (B) Idealized topography of the cortical parcellation units (uppercase). Subcortical VOIs and the cerebellum are not shown. For the mean volume and number of pixels for each VOI, as well as abbreviations and refinements, see the Method section and Table 1. (i.e., the anterior division of the cingulate cortex [CGa; BA 32], the paracingulate gyrus [PAC; BAs 24 and 33], and the supplementary motor cortex [SMC; BA 4]) and two parietal VOIs (the posterior division of the cingulate cortex [CGp; BA 23] and the precuneus [PCN; BAs 7 and 31]). Furthermore, three VOIs were defined along the intrasylvian cerebral surface (i.e., the total extent around the Sylvian fissure [sy], the Heschl's gyri [he], and the insular cortex [INS]). Finally, the cerebellar hemispheres $(\mathrm{CH})$ were defined as a broad VOI, and the caudate nuclei (cau), the putamen (pu), and the thalamus (thal) were defined as subcortical structures. The latter VOIs (he, INS, $\mathrm{CH}$, cau, pu, and thal) are not visible on the cortical surface shown in Figure 2. The interrater reliability of this VOI-defining procedure between two staff members was evaluated in our previous study (Jaeggi et al., 2003) and ranged between $r=$ .82 and $r=.93$, reflecting a high degree of reliability, also being in accordance with values reported by Caviness, Meyer, Makris, and Kennedy (1996).

For each VOI, activation changes with respect to the different levels of load, as compared with the baseline, were evaluated separately for both performance groups and hemispheres. Since we were interested in interindividual differences in neural activation based on performance, we calculated correlations (Pearson's) between the BOLD response and the performance measures $\left(P_{\mathrm{r}}\right)$ in all VOIs at the various levels of load, in order to obtain an estimate of the relationship between brain activity and behavior. Nonparametric bootstrapping with $R=9,999$ replicates of Fisher's $z$ transformation at the normal 95\% confidence interval were applied in order to estimate reliability - that is, to test the null hypothesis of zero correlations. For those VOIs for which significant activation changes were observed and for which a significant brain-behavior correlation was observed, two-way repeated measures ANOVAs were calculated with task load (1-back to 3-back) as a within-subjects factor and performance group (low and high performer) as a between-subjects factor. Post hoc $t$ tests (twotailed) were administered in case of significant interactions and were Bonferroni corrected for multiple comparisons.

\section{RESULTS}

\section{Behavioral Data}

Generally, the participants performed very well in all the tasks. Even at high levels of load, several participants were still able to perform the task adequately, but as was predicted, accuracy levels were near chance level in the most difficult condition (3-back dual task) in some participants. In this condition, the median split (3-back; $P_{\mathrm{r}}$ ) was applied in order to differentiate the two groups of participants [low-performing participants, $P_{\mathrm{r}}<.48(\bar{x}=$ $0.38, S D=0.04)$; high-performing participants, $P_{\mathrm{r}}>.48$ $(\bar{x}=0.64, S D=0.04)]$.

The two-way repeated measures ANOVA indicated a significant main effect of load-that is, 0-back to 3-back tasks [accuracy, $F(1.75,14)=173.43, p<.001$; RT, $F(1.82,14)=30.62, p<.001]$ - and a significant main effect for performance group in RT $[F(1,14)=10.47, p<$ $.01]$. A significant interaction between load and performance group was observed for accuracy $[F(3,4)=16.80$, $p<.001]$ and RT $[F(3,4)=3.81, p<.05]$. Post hoc tests for accuracy yielded significant differences between the two performance groups at the 3-back level only, where it was expected, since this was the group assignment criterion $[t(13)=4.90, p<.001]$. In RT, group differences were observed at the 1-back, 2-back, and 3-back levels, in all of which the high-performing group was responding significantly faster [1-back, $t(13)=2.25, p<.05$; 

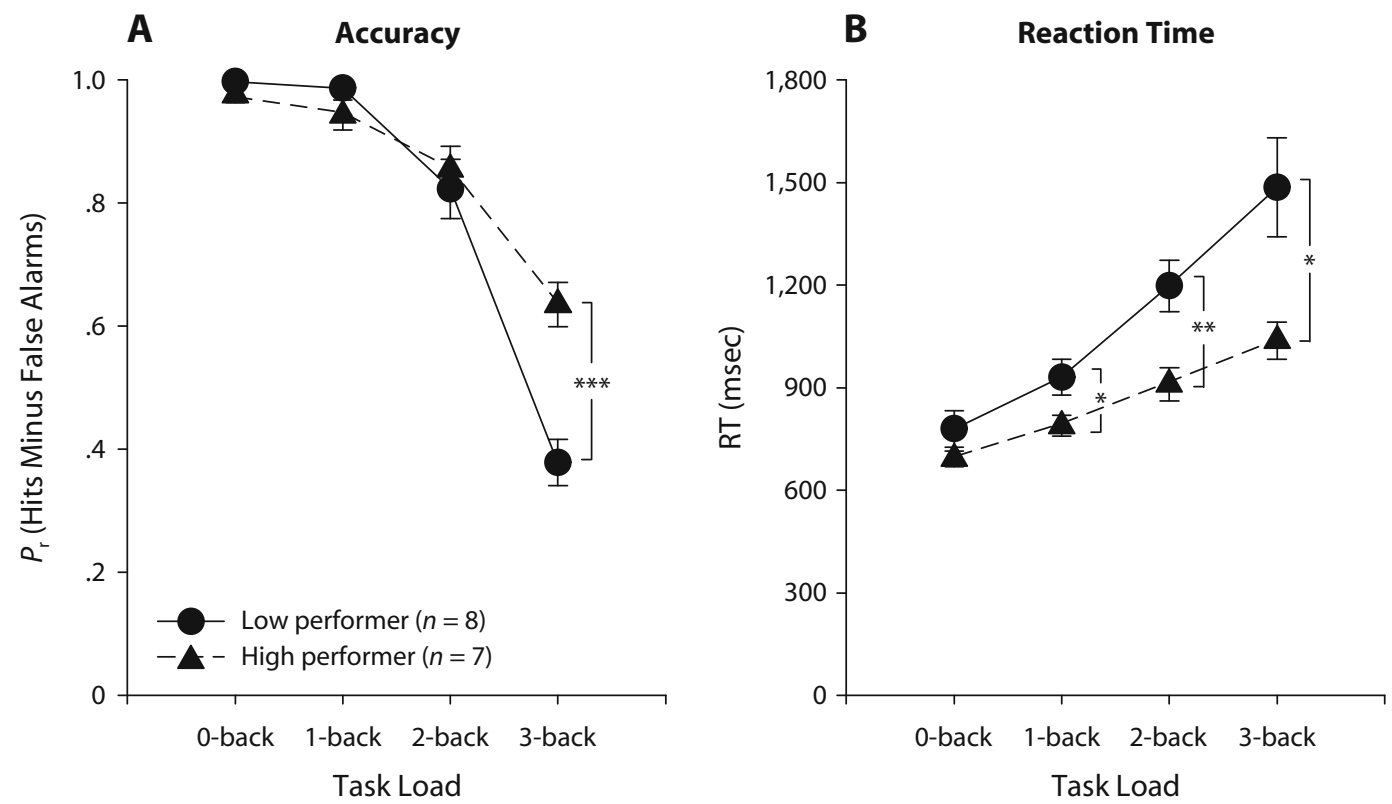

Figure 3. Means and standard errors of the mean (SEMs) for the two groups of participants for each level of load, plotted as mean between the auditory and the visuospatial conditions. (A) Accuracy $\left(P_{r}\right.$, i.e., hits minus false alarms). (B) Reaction times (in milliseconds; hits only). Differences between groups are indicated $\left({ }^{*} p<.05 ;{ }^{* *} p<\right.$ $\left..01 ;{ }^{* * *} p<.001\right)$.

2-back, $t(13)=3.12, p<.01 ; 3$-back, $t(13)=2.74, p<$ $.05]$. Descriptive data for both behavioral measures are shown in Figure 3.

In order to investigate whether there where performance differences between the visuospatial and the auditory $n$ back tasks, a trade-off measure was calculated as difference between the two tasks at each level of load, where zero would represent perfectly equal performance between the two task-modalities. For the whole sample, this trade-off index differed significantly from zero only in the 2 - and 3-back tasks [0-back, $t(14)=1.01$, n.s.; 1-back, $t(14)=$ 1.87, n.s.; 2-back, $t(14)=2.47, p<.05$; 3-back, $t(14)=$ $2.56, p<.05$ ], indicating that the participants were able to perform both tasks equally well at the easier task levels but gave priority to one- that is, to the visuospatial task at the 2- and 3-back levels - despite being instructed to pay attention to both tasks and despite their general statement of not having given priority to one task. The low-performing participants had significant trade-off values in the 1-back $[t(7)=2.39, p<.05]$ and the 2-back $[t(7)=2.68, p<$ .05] tasks, giving priority to the visuospatial task, to the detriment of the other, at intermediate levels, whereas the high-performing group expressed this difference only at the 3-back level $[t(6)=6.14, p=.001]$, also preferring the visuospatial task. Significant group differences in this trade-off measure were observed only at the 3-back level $[t(13)=-2.43, p<.05]$.

Reliability measures for $P_{\mathrm{r}}$ were low for the easier tasks, presumably due to ceiling performance ( 0 -back, $r=-.03$; 1-back, $r=.03$; n.s.), but were in the acceptable range for the more difficult tasks (2-back, $r=.43, p=$ n.s.; 3-back, $r=.73, p<.01)$. There were no significant differences between practice and fMRI sessions at any level of load [0-back, $t(14)=0.38 ; 1$-back, $t(14)=-0.83$; 2-back, $t(14)=-1.30 ; 3$-back, $t(14)=-1.71$; all n.s.).

The qualitative analysis of the posttest inventory revealed that most of the participants used a visual-tracking strategy for the visuospatial task and a verbal rehearsal strategy for the auditory task - that is, combining the two strategies, since it was a dual-task situation. However, most of the participants used more than these two strategies - that is, changing between strategies as the task demand increased (mean used strategies: 1-back, $\bar{x}=$ 2.33, $S D=0.49$; 2-back, $\bar{x}=2.73, S D=1.39$; 3-back, $\bar{x}=3.27, S D=1.71)$. Interestingly, however, the highperforming participants mentioned the use of fewer explicit strategies than did the low-performing group, with the mean number of reported strategies for the high performers being $2.14(S D=0.57)$ for all levels of load versus $3.33(S D=$ 1.25 ) in the low-performing group [difference between high and low performers, $t(10.10)=2.42, p<.05$, two-tailed]. Some of the high-performing participants stated that they had not relied on strategies but, rather, experienced a kind of intuitive problem solving and relied on automatic processes, whereas the low-performing participants stated that they combined and tried out different strategies, changing them frequently and unsystematically during task execution.

\section{Imaging Data}

The activation data were evaluated for all 30 VOIs. Activation changes with respect to the different levels of load, as compared with the baseline, for both performance groups and hemispheres are shown separately in Table 2. However, the activation changes for those VOIs for which 


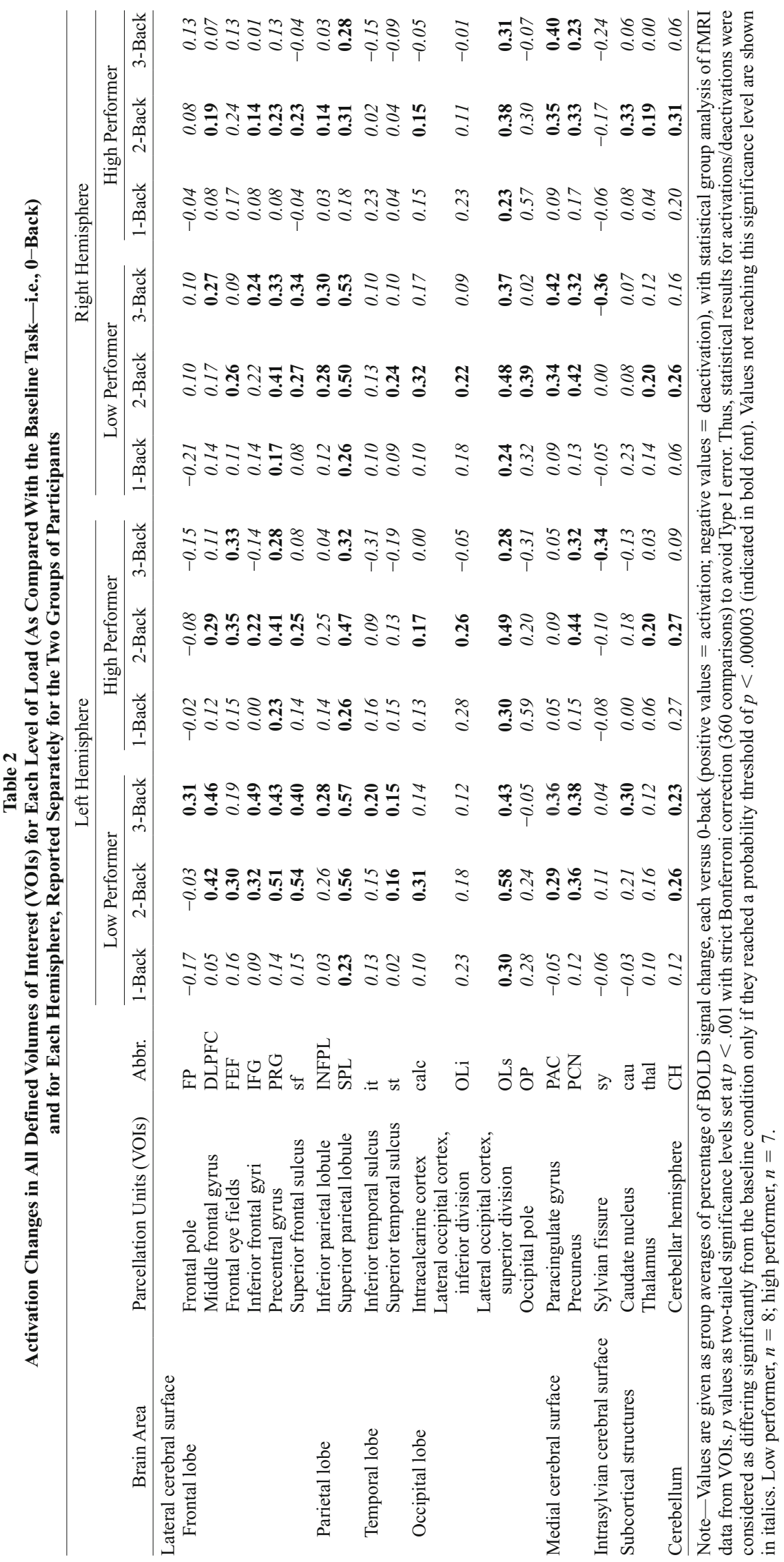


no significant activation change was observed in any condition are not reported in Table 2 (i.e., SFG, ce, POG, TP, $\mathrm{SMC}, \mathrm{CGa}, \mathrm{CGp}$, he, INS, and pu).

\section{Brain-Behavior Correlations}

The correlations between the BOLD response and the performance measures were not significant in any VOI at the 1-back level, with values ranging between $r=-.47$ and $r=.48$. Similarly, no significant correlations were observed at the 2-back level, except for the right thalamus, where a substantial positive correlation was observed $[r=$ $.73, p<.01 ; P(-0.38 \leq 0.73 \leq 0.81)]$. In the 3 -back task, significant correlations were observed in seven VOIs, all of them being negative and observed mostly in the left hemisphere. In frontal regions, correlations were significant in the left DLPFC $[r=-.53, p<.05 ; P(-1.28 \leq-0.53 \leq$ $0.27)]$, in the left IFG $[r=-.66, p<.01 ; P(-1.28 \leq$ $-0.66 \leq-0.17)]$, and in the right sf $[r=-.52, p<.05$; $P(-0.98 \leq-0.52 \leq-0.10)]$. Temporally, there were significant correlations in the left st and left it gyri [st, $r=$

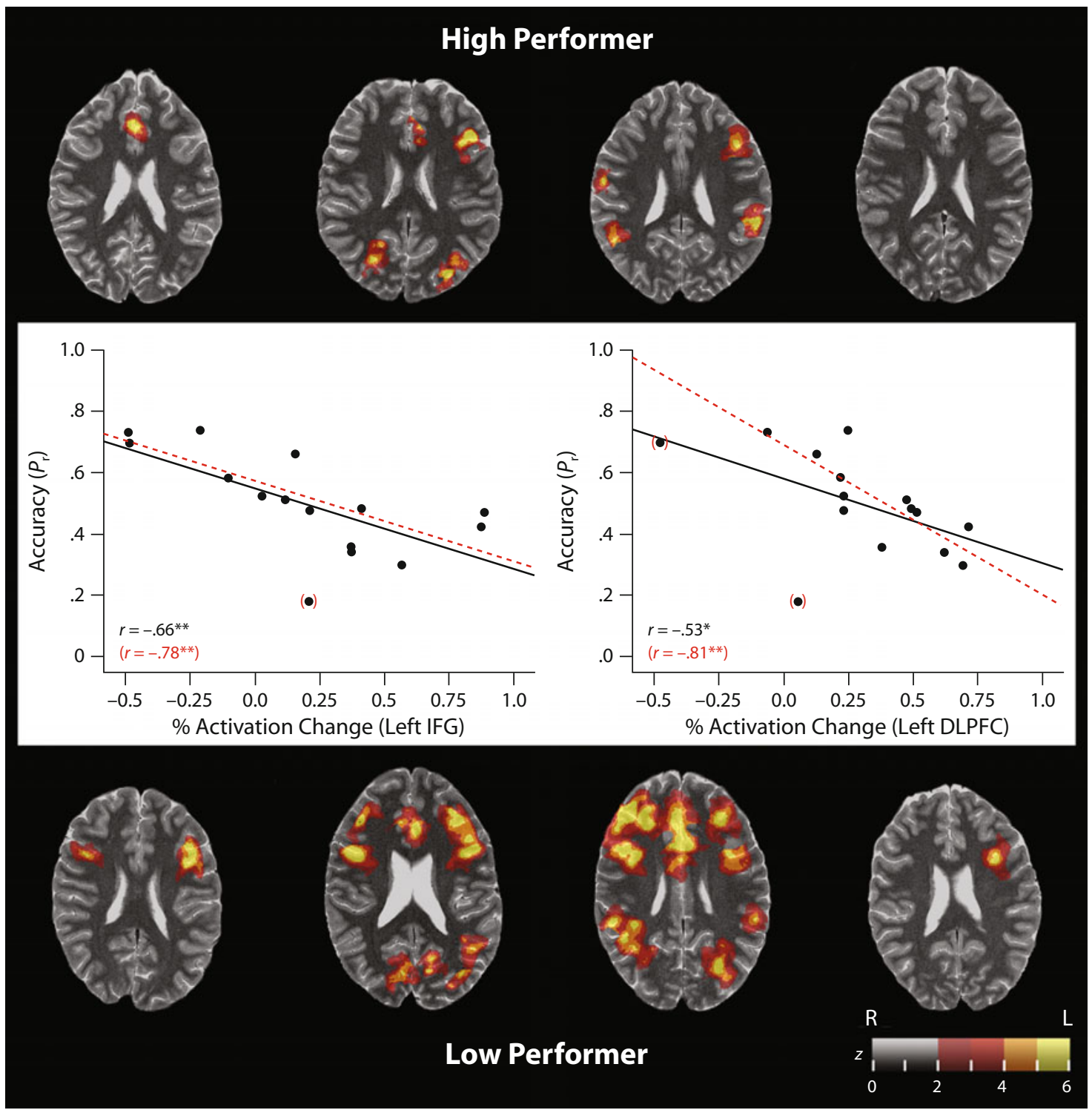

Figure 4. fMRI activation of the 3-back versus 0-back contrast, shown for the four highest and the four lowest performing participants, ordered by performance from left to right (top four panels, best performers; bottom four panels, worst performers). To show the $z$ score maps of a representative part of the brain on just one panel each, a maximum intensity projection of five adjacent functional slices was overlaid onto one structural slice. Clusters containing a $z$ score of at least $5.0(p<.05$, Bonferroni corrected for multiple comparisons) were included, with their extent color coded down to a $z$ score of $2.0\left(p<.05\right.$, uncorrected). In the center of the figure, scatterplots of accuracy $\left(P_{\mathrm{r}}\right)$ versus BOLD response in the left dorsolateral prefrontal cortex (DLPFC) and the left inferior frontal gyrus (IFG) are depicted for the 3-back task, indicating that, with increasing accuracy, the BOLD response decreases, as expressed by the negative correlations. The correlation coefficients after removal of outliers (in parentheses) are indicated in red and in parentheses, and the corresponding regression line is shown as the dotted red line. ${ }^{*} p<.05 .{ }^{* *} p<.01$. 
$-.57, p<.05, P(-1.12 \leq-0.57 \leq-0.14)$; it, $r=-.60$, $p<.01, P(-1.27 \leq-0.60 \leq-0.08)]$. Furthermore, significant negative correlations were observed in the right calc $[r=-0.56, p<0.05 ; P(-1.08 \leq-0.56 \leq-0.09)]$ and in the left cau $[r=-.53, p<.05 ; P(-1.18 \leq-0.53 \leq 0.06)]$. Two examples of the observed negative correlations (left DLPFC and left IFG) are depicted in Figure 4.

For those VOIs for which significant brain-behavior correlations were observed (i.e., left DLPFC, IFG, st, it, and cau; right calc, sf, and thal; see Table 3), two-way repeated measures ANOVAs with load (1-back to 3-back) as the within-subjects factor and performance group (low and high performer) as the between-subjects variable were calculated, and the results are summarized in Table 3.

\section{Main Effects}

Main effect of load. A main effect of load was observed for every VOI; however, three different patterns of activation could be differentiated. (1) Load-dependent activation increases - that is, increases from 1- to 2-back and/or from 1- to 3-back-were observed in frontal regions (DLPFC, IFG, and sf) and, to some extent, in the cau. After 2-back, however, the activation leveled off. That is, the activations between the 2- and the 3-back conditions did not increase further; rather, they showed a tendency to decrease (e.g., in the sf), although the difference between these two load conditions was not statistically significant. (2) An inverted U-shaped (or rather, an inverted V-shaped) pattern - that is, a significant increase from 1- to 2-back and a decrease from 2- to 3-back conditions, without difference between the 1- and the 3-back conditions-was observed in the calc and in the thal. (3) In temporal regions (i.e., in the area surrounding the st and the it sulci [st, it]), a significant load-dependent activation decrease - that is, a decrease from 1- to 3-back and from 2- to 3-back-was observed. The difference between 1- and 2-back levels was not significant in these VOIs. The mean activation changes depending on various levels of load and grouped according to the three activation patterns are depicted in Figure 5A.

Main effect of performance group. A main effect of performance group was observed in every VOI. The low-performing group consistently and reliably expressed higher activations than did the high-performing group.
Interaction (load $\times$ performance group). This interaction was significant for every VOI, with the exception of the thal. Post hoc tests (all two-tailed and Bonferroni corrected for multiple comparisons) showed that group differences were observed at highest levels of load (3-back) in every VOI, and also at the 2-back level in the posterior VOI (calc). No difference between groups was observed at the 1-back level in any VOI. As has already been indicated by the main effect of performance group, the low performers had larger activations at higher levels of load, as compared with the high-performing group. Looking at the load-dependent activation patterns for each performance group separately, a comparable activation pattern was observed in the three prefrontal VOIs (DLPFC, IFG, and sf): Whereas the low-performing group showed an activation increase, the high-performing group showed the inverted U-shaped pattern. Although the activation in the low-performing group did not significantly increase from the 2- to the 3-back level, it was highest at the 3-back level. In temporal (st and it) and posterior (calc) regions, as well as, to some extent, in the basal ganglia (cau), the highperforming group showed activation decreases, whereas the low-performing group responded with activation increases (calc, cau, and st) or no difference between load levels (it). The mean activation changes depending on various levels of load and performance group and arranged according to the main activation patterns referenced above are depicted in Figure 5B.

\section{DISCUSSION}

With the parametric dual task used in this study, we observed a load-dependent activation pattern in every defined VOI that showed brain-behavior correlations. In accordance with the results of our prior study (Jaeggi et al., 2003), an increase in activation was observed in lateral prefrontal areas (DLPFC, IFG, and sf) and, to some extent, in the basal ganglia. This pattern can be interpreted as reflecting the executive and control processes necessary to fulfill the task demands (Jansma, Ramsey, Slagter, \& Kahn, 2001; Owen et al., 2005) but, also, the mental effort that is engaged as task difficulty increases (Duncan \& Owen, 2000; Frith \& Dolan, 1996). The (subjectively

Table 3.

Main Effects and Interactions of the Two-Way ANOVA Shown for Each Defined Volume of Interest (VOI) in Which Significant Activation Changes and Brain-Behavior Correlations Were Observed

\begin{tabular}{|c|c|c|c|c|c|c|c|c|c|}
\hline \multirow[b]{2}{*}{ Brain Area } & \multirow[b]{2}{*}{ Parcellation Units (VOIs) } & \multirow[b]{2}{*}{ Abbr. } & \multirow[b]{2}{*}{ Hemisphere } & \multicolumn{2}{|c|}{ Load } & \multicolumn{2}{|c|}{ PG } & \multicolumn{2}{|c|}{ Load $\times$ PG } \\
\hline & & & & $F$ & $p$ & $F$ & $p$ & $F$ & $p$ \\
\hline \multirow[t]{3}{*}{ Frontal lobe } & Middle frontal gyrus & DLPFC & left & 26.05 & $* * *$ & 19.84 & $* * *$ & 14.42 & $* * *$ \\
\hline & Inferior frontal gyri & IFG & left & 8.57 & *** & 37.58 & *** & 15.66 & $* * *$ \\
\hline & Superior frontal sulcus & sf & right & 15.90 & *** & 28.29 & $* * *$ & 9.34 & $* * *$ \\
\hline \multirow[t]{2}{*}{ Temporal lobe } & Inferior temporal sulcus & it & left & 6.05 & $* *$ & 12.31 & $* * *$ & 10.49 & $* * *$ \\
\hline & Superior temporal sulcus & st & left & 7.45 & *** & 4.93 & $*$ & 15.33 & $* * *$ \\
\hline Occipital lobe & Intracalcarine cortex & calc & right & 9.99 & *** & 12.10 & $* * *$ & 6.62 & $* * *$ \\
\hline \multirow[t]{2}{*}{ Subcortical } & Caudate nucleus & cau & left & 4.38 & $*$ & 10.29 & $* * *$ & 6.91 & $* *$ \\
\hline & Thalamus & thal & right & 8.89 & *** & 6.77 & $* *$ & 1.25 & n.s. \\
\hline
\end{tabular}

Note-PG, performance group; $N=630$ (number of measurements); $d f=1$ for main effect of PG; $d f=2$ for main effect of load and for the load $\times \mathrm{PG}$ interaction. ${ }^{*} p \leq .05 .{ }^{* *} p \leq .01 .{ }^{* * *} p \leq .001$. 
A Left DLPFC

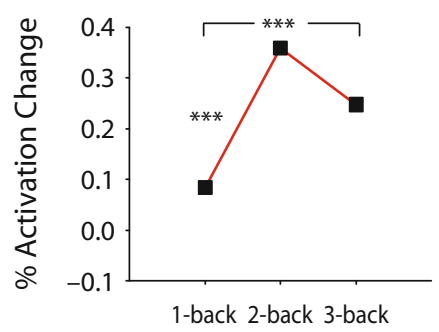

Right calc

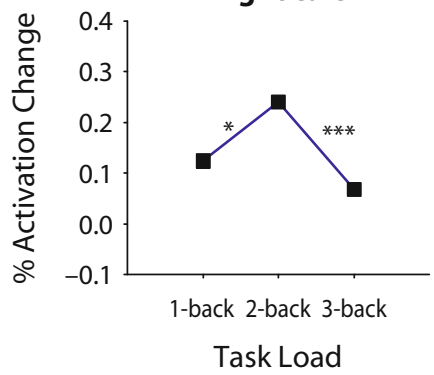

B Left DLPFC

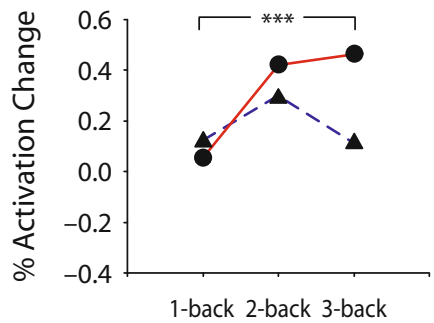

Right calc

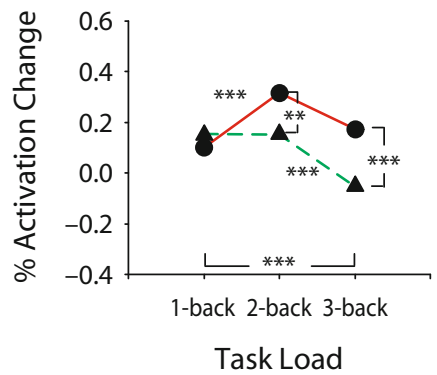

Left IFG

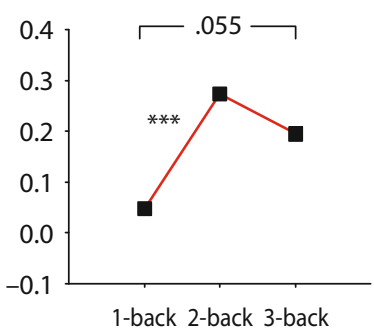

Right thal

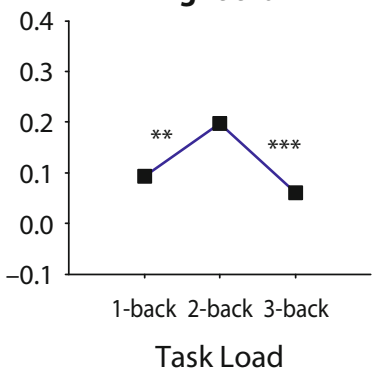

Left IFG

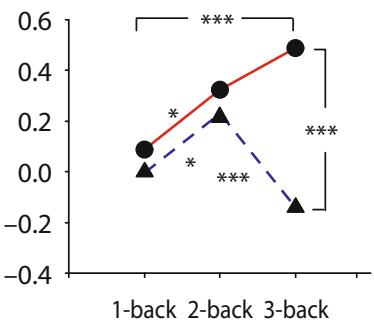

Left cau

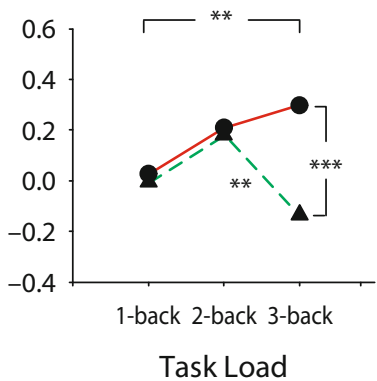

Right sf

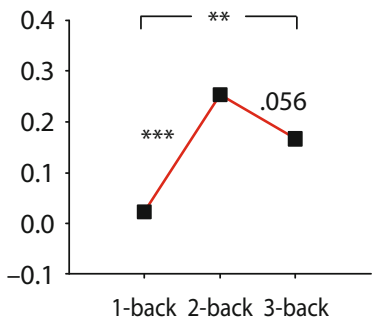

Left st

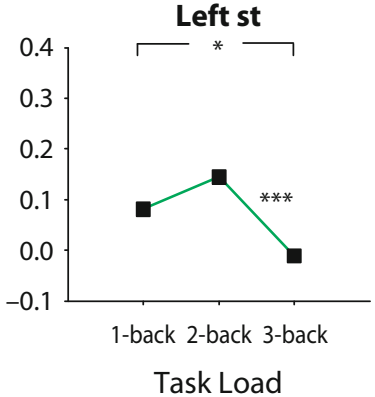

Right sf

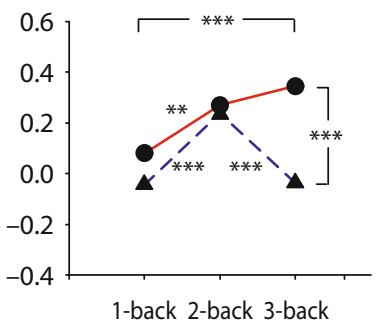

Left st

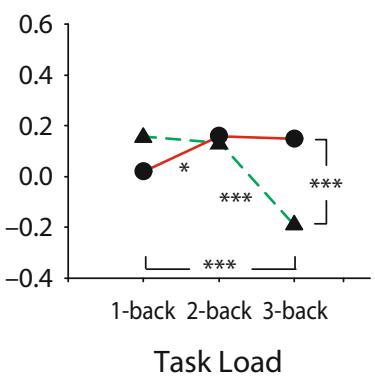

Left cau

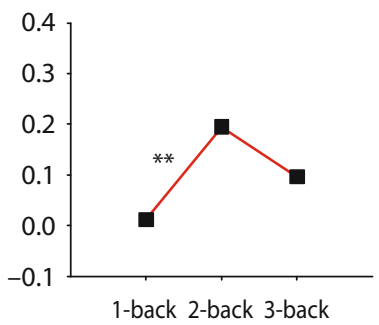

Left it

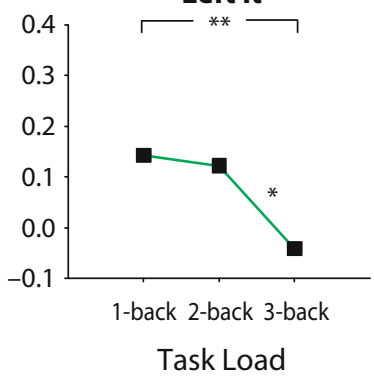

Task Load

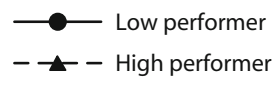

Figure 5. (A) Significant main effects of load. (B) Significant interactions (load $\times$ performance group), to visualize individual differences. Represented are the mean activation changes in response to the three load conditions for each volume of interest, ordered by their activation patterns. Increases: red lines (increase from 1- to 3-back and/or 1- to 2-back). Inverted U-shaped curve: blue lines (increase from 1- to 2-back and decrease from 2- to 3-back). Decreases: green lines (decrease from 1- to 3-back and/or from 2- to 3-back). No load-dependent activation change: black lines. Significant differences between the various load conditions and between groups are indicated $\left({ }^{*} p<.05 ;{ }^{* *} p<.01 ;{ }^{* * *} p<.001\right.$; all corrected for multiple comparisons). DLPFC, dorsolateral prefrontal cortex; IFG, inferior frontal gyrus; sf, superior frontal sulcus; cau, caudate nuclei; calc, intracalcarine cortex; thal, thalamus; st, superior temporal sulcus; it, inferior temporal sulcus.

perceived) demands at the two higher load levels might be comparable, thereby resulting in the asymptotic pattern of activation in these VOIs. The activation increases were observed to be mainly left lateralized (DLPFC and IFG), being in accordance with findings in the literature reporting that left-lateralized activation in the PFC occurs when evaluative processes are engaged in retrieval pro- cesses (e.g., Mitchell, Johnson, Raye, \& Greene, 2004; Prince, Daselaar, \& Cabeza, 2005; Ranganath, Johnson, \& D'Esposito, 2000), which are essential for specifying the source of a memory (Johnson, Hashtroudi, \& Lindsay, 1993). Therefore, the left PFC seems to be recruited specifically during memory tasks that demand specific source attributions - processes that seem to be critical for 
carrying out an $n$-back task at higher levels of load. It has been further suggested that the left PFC is engaged in the processing of sequential dependencies between single adjacent events (Wood \& Grafman, 2003), which also seems relevant for such type of tasks. Research on dividedattention tasks (Nebel et al., 2005) has provided further evidence that there is a shift to left-lateralized processing if a task is executed as a dual task, instead of a single task, or when the attentional load is increased. Conversely, the load-dependent activation increases observed in the right sf could reflect memory processes based on recency judgments (Dobbins, Rice, Wagner, \& Schacter, 2003; Mitchell et al., 2004), which might also be involved in the task used in the present study. Since the activation showed a tendency to decrease from 2- to 3-back in this VOI, it may be that this recency-based strategy plays a smaller role in the 3-back task, since more (interfering) items have to be processed in the course of the task and participants might rely increasingly on evaluative processes, as was discussed above. Similarly, at the 1-back level, the task is less dependent on familiarity, since participants still "know" the items that were presented just $2.5 \mathrm{sec}$ earlier, without interfering stimuli in between.

In two areas, the posterior VOI (calc) and the thal, the activation pattern was inverted U-shaped and might reflect capacity limitations, as is stated in the hypothesis formulated by Callicott et al. (1999) and D'Esposito (2001) in regard to the PFC, which is also supported by the findings of other authors for more posterior areas (Linden et al., 2003; Todd \& Marois, 2004; Xu \& Chun, 2006). In occipital regions such as the calc, activation decreases are often observed with perceptual-related and/or priming-related facilitation processes (Schacter \& Buckner, 1998). Again, such processes may be involved more in easy or difficult tasks, but not in tasks of intermediate difficulty (2-back), where more elaborate strategies - as, for example, rehearsal and explicit updating processes - can be applied: In the easier version of the task (1-back), strategies may be involved to a lesser degree, and participants may rely more on automatic processes; in the difficult version (3-back), strategies such as rehearsal may not be appropriate either, since too many items will have to be remembered, thus exceeding the WM capacity limit. Consequently, more automatic processes may be involved at the 1- and 3-back levels than at intermediate levels, which is reflected in the lower activations. That higher activations represent a shift from automatic to controlled processing is also supported by the findings of other authors (e.g., Jansma et al., 2001). Regarding the thal, the interpretation is more difficult, especially as to the right-hemispheric activations. Egner and Hirsch (2005) have reported right-lateralized activations in the thal in response to negative priming, and therefore, processes similar to those discussed above in relation to the calc might also apply to the thalamic activation found here.

In lateral temporal regions (st and it), a load-dependent decrease was observed, suggesting that the involvement of these regions in load-dependent processing is different from that of the prefrontal, posterior, and subcortical areas. The lateral temporal regions are assumed to be involved in visual-encoding processes, object perception, and auditory word recognition, which are more commonly observed left lateralized (for a review, see Cabeza \& Nyberg, 2000). Therefore, the load-dependent deactivations in the left lateral temporal cortices in our study indicate that these regions are more involved in the control task (0-back), which is clearly perceptual and not strongly related to memory processes. Furthermore, as is indicated by the trade-off measures, priority was given to the visuospatial task as difficulty increased. Thus, the temporal regions, which are probably more involved in the auditory version of the task, might have been used to a lesser extent with increasing load. Together with the prefrontal activation pattern, our results imply that the higher the memory load, the more prefrontal areas are engaged, suggesting a shift from perceptual to WM processes.

Yet the main goal of this study was to investigate the differential effects of WM load on activation patterns in various cortical areas, using an interindividual-differences approach. Our results showed clear and reliable differential behavioral dissociations between performance groups, which went along with differential activation patterns that went beyond the patterns discussed for the whole sample. In contrast to our expectations, the high-performing group showed lower activations than did the low-performing group, especially pronounced at higher levels of load, indicating that there are some cortical areas that "keep cool" in order to lead to excellent performance. There are reports of overall lower activation in high-performing groups than in low-performing groups (Rypma, Berger, \& D’Esposito, 2002) or, more specifically, in the DLPFC (Rypma \& D'Esposito, 1999, 2000; Smith et al., 2001) and also in other brain regions, as, for example, in hippocampal structures (Bosshardt et al., 2005). However, in the study by Rypma et al. (2002), in which memory load was manipulated, the high-performing participants showed activation increases with increasing processing demand in the lateral PFC, whereas the low-performing participants showed higher activation but minimal load-dependent increase. In contrast, the low-performing group in our study consistently showed load-dependent activation increases in almost every VOI. We therefore suggest that the higher activation changes in this group result from processes that compensate for decreasing performance rates. Such activation patterns might relate to the findings in the literature on aging, where additional brain areas are usually activated in the elderly, as compared with young participants, which has been discussed in terms of compensatory processes taking place (e.g., Cabeza et al., 1997) and which led to the proposal of the HAROLD model (Cabeza, 2002). In contrast to the compensation view, it has been suggested that the recruitment of additional brain areas, especially the decrease of lateralization, could be interpreted rather in terms of a failure to engage specialized neural mechanisms (dedifferentiation hypothesis; see, e.g., Li \& Lindenberger, 1999). In this view, lower performance should be accompanied by smaller tradeoff measures in the behavioral task, and consequently, a less lateralized activation pattern would be expected in the low-performing group. This was clearly the case for 
the behavioral measure at the highest load level (3-back), where the high-performing group had larger trade-off values, but the imaging data are less supportive for this view: The low-performing group generally had larger activation changes than did the high-performing group, especially at higher levels of load, but there was no evidence for a larger lateralization in the high-performing group that could be associated with their superior performance. Still, no clear differential pattern between prefrontal and other VOIs was observed in the low-performing group, as compared with the high-performing group, reflecting an undifferentiated way of processing and providing some evidence for the dedifferentiation view. To put it differently, the high brain activity of low performers observed in the most extreme mental load condition either is not relevant or impairs performance, an interpretation that is compatible with the neural efficiency hypothesis, as recently discussed by Neubauer, Grabner, Fink, and Neuper (2005) or initially described by Haier et al. (1992).

In contrast to the widespread load-dependent activation increase in the low-performing group, the highperforming group showed differential activation patterns between VOIs: Whereas an inverted U-shaped pattern was observed in prefrontal areas (left DLPFC, IFG, and right sf), load-dependent decreases were expressed in the posterior and temporal regions, as well as, to some extent, in the basal ganglia (right calc, left st, it, and cau), providing evidence for a differential involvement of prefrontal and posterior regions in load-dependent processing in this group. The question now arises as to how such differential activation patterns could be interpreted. The inverted U-shaped curve observed in prefrontal areas certainly does not seem to reflect "giving up" or disengaging from the task, as was suggested by Callicott et al. (1999) and D'Esposito (2001). Rather, our high-performing participants showing this pattern still tried to fulfill the task demands and even succeeded, as the behavioral data way beyond chance level indicate. It is important to note that the low-performing participants also still engaged in the task, which is reflected in their subjective reports, as well as in the performance rate, which was near but still above chance level; therefore, contrary to Callicott et al. (1999) the inverted U-shaped curve did not emerge in our lowperforming group. It is evident that the prefrontal inverted U-shaped curve observed in the high-performing participants does not support the idea that processing at capacity limitations is being reflected and, consequently, that the task has been disengaged from but may represent a shift of strategies as the task got more difficult. Indeed, differences in the use of strategies were reported by the two participant groups: The high-performing participants reported the use of fewer strategies than did the low-performing group, and they also showed fewer changes in strategies applied in response to the WM load, whereas the low-performing group seems to have used and tried out more strategies with increasing task difficulty, which might account for the higher activations in the PFCs (and maybe, also, in other VOIs) in this group. Some evidence for that interpretation has been provided by several studies by Bor and colleagues (Bor, Cumming, Scott, \& Owen, 2004; Bor \&
Owen, 2007). In their studies, prefrontal activity was associated with elaborative strategic processes, rather than with task difficulty, since they observed increases in prefrontal activation with the use of strategies, along with a lower WM demand and a better performance. In our study, however, better performance was associated with lower activation, but the difference might still lie in the strategies used or in the type of processing: The high-performing group reported using more "intuitive" problem-solving strategies and relying on automatic processing, rather than using resource-consuming strategies, and consequently, the activation decreased. However, the U-shaped curve in this group still might represent subjectively perceived capacity limitations, which would motivate a shift of strategies: In the 2-back task, where the difference between the groups was not significant in most areas, it may be that the same processes as rehearsing the four items (since it was a dual task) took place in both groups. The capacity limit of the attentional or WM focus has been defined as four items by Cowan (2001), and it seems that both groups were able to process these items with the same success and with comparable strategies (i.e., rehearsal), which was also reflected by an accuracy rate of over $80 \%$ in both groups. It is at the highest level of load - that is, at remembering and updating six items every $3 \mathrm{sec}$ - that the difference becomes evident, and it is at that level that the differential activation patterns reflect individual differences in processing at supracapacity levels. Taking the subjective reports of the strategies used into account, our data provide enough evidence that the prefrontal inverted U-shaped curves in the high-performing group might represent a shift from controlled to automatic processing, as described above (Jansma et al., 2001), when these participants came to the difficult task. The activation decreases in the temporal VOIs might similarly reflect this shift in processing and strategy use; however, there might be a differential effect from perceptual to more internal processes. It seems, therefore, that in the high-performing group, there were VOIs that responded differentially to processing at capacity limits. In contrast, the data suggest that the low-performing participants were impaired in recruiting the optimal brain regions in an effective mode, since they seem to have chosen too many and less effective strategies, which was also reflected in the longer RTs.

It can therefore be concluded that increased activity in this task might not represent task difficulty per se, as described by Duncan and Owen (2000), but, rather, the use of effort-demanding strategies. Some recent findings by Vogel, McCollough, and Machizawa (2005) also support this point: In their electrophysiological study, participants with low WM capacity had more difficulty differentiating between relevant and irrelevant stimuli, which went along with higher activations. It is well agreed on (e.g., Jonides $\&$ Nee, 2006) that key determinants of WM performance are selectivity and resistance to interference. In our task, every item had to be processed and updated very quickly in WM, and it had to be decided every $3 \mathrm{sec}$ whether the item was a potential target or not. If the low-performing participants tried to process and remember every item and did not succeed in "dropping" the oldest ones efficiently, 
thereby adding to the memory load, it is easily comprehensible that many resources and brain areas were used in order to fulfill these demands. High-performing participants, in turn, seemed to very effectively update their WM contents, especially at higher levels of load, which was, in turn, reflected in decreasing activation.

Alternatively, the higher activation changes in the lowperforming group may reflect monitoring of failure, rather than WM load or the use of strategies. In the literature, there is evidence that medial prefrontal and bilateral inferior parietal regions are involved in error detection (e.g., Garavan, Ross, Murphy, Roche, \& Stein, 2002; Rubia, Smith, Brammer, \& Taylor, 2003), and therefore, lowperforming participants should differentiate primarily in these VOIs. However, the activation differences were observed in a more widespread network, and thus, an interpretation of the higher activations based solely on failure monitoring and error detection would not be exhaustive. Moreover, the brain-behavior correlations were not significant in those VOIs, where error detection processes might be expected in the first place.

It can be asked, though, what would happen if highperforming participants were also confronted with a task that exceeded their capacity limit. The behavioral data indicate that the high-performing participants had not yet reached their capacity limits, and conceivably, large activation changes similar to those of the low-performing participants could have occurred also in this group had even higher task demands been used (e.g., 4- and 5-back tasks), reaching or exceeding their capacity limits. Alternatively, high-performing participants possibly use qualitatively different and more efficient strategies - that is, keeping "cool brains" when confronted with highly demanding tasks, regardless of their performance level. Thus, for the high-performing participants, the activation changes might not have increased, even at higher levels of load. Still, the question remains why the high-performing participants showed (high) activations at the 2-back level similar to those of the low-performing group in most VOIs. It could well be that this group "normally" shows high activation changes at low and intermediate levels of difficulty but that, in the face of a challenge, they switch to a different and more efficient processing mode that does not lead to high activation changes. It seems more likely that effective functional connectivity, resulting in low overall signal changes, might account for the high performance in this group, which was not investigated in this study. The consistent negative correlations between the BOLD signal and the accuracy measures in the 3-back task provide further evidence that there is a whole network of areas that play a role only if participants are struggling with a task - that is, only at the supracapacity level. They also may be interpreted as evidence against a qualitative difference between high- and low-performing groups at this load level; rather, the better the performance, the fewer the demands that are placed on the areas involved, which is gradually expressed over all participants (see also Callicott et al., 2000). This finding can be related to training studies, which usually have reported an activation decrease in task-related cortical brain regions after the task has undergone substantial practice. Such practice-related activation decreases have been demonstrated, for example, with motor learning (van der Graaf, de Jong, Maguire, Meiners, \& Leenders, 2004) and procedural learning (Kassubek, Schmidtke, Kimmig, Lucking, \& Greenlee, 2001), but also with tasks relating to higher cognitive functions, such as WM (Jansma et al., 2001) or mathematical problem solving (Qin et al., 2004). Possibly, some participants are faster at learning a task such as the $n$-back task used and, therefore, rely on already established neuronal circuits in the fMRI-session, which is expressed in a lower BOLD signal in task-related brain areas and in interindividual differences such as those observed here. In other words, the high activation in the low-performing group may have reflected ongoing learning - that is, brain plasticity at work. In accordance with the training literature, it is entirely possible that high activation changes in low-performing participants would also decrease if they did not get enough practice with the task.

In sum, it seems that there is an overlapping WM- and attention-related neural network that mediates performance at supracapacity levels and is differentially involved in good and bad performance. Thus, inverted U-shaped curves and increases or decreases in activations are not easy to interpret without taking individual performance and the use of strategies into account, adding to the importance of interpreting neuroimaging data from the viewpoint of interindividual differences, especially if difficult tasks such as the dual task applied in this study are used.

The differential activation pattern of good and bad performers in the present study clearly shows that brain activation, cognitive load, and performance are not related in a simple and linear way. Activation discriminates high- from low-performing individuals but, nevertheless, seems to be related to performance only indirectly, which can be observed differentially in various VOIs and performance groups. Overall, the brain of good performers "keeps cool" in terms of brain activity in conditions of extreme cognitive demand. There seem to be no brain areas compensating with a higher activation in high-performing participants, but there was a consistent pattern of low activation across the whole brain in this group, most consistently pronounced at high levels of load (see Table 2), which might be explained in terms of a successful, task-adequate, and efficient functioning of neural circuits.

\section{CONCLUSION}

Our data show that there are large and reliable interindividual differences in processing at capacity limits, which can be observed on both a behavioral and a neuronal basis. These differences most likely result from more efficient utilization of resources especially pronounced in highperforming participants, which also show differential activation patterns in prefrontal and posterior areas, in contrast to the low-performing group, which consistently expresses load-dependent activation increases over most VOIs. From the present data, it is not clear why individual participants 
differ in efficiency, the application of appropriate strategies, and the differentiation of relevant and irrelevant information. It is also an open question as to whether participants can be taught to be more efficient with appropriate training (see, however, Jaeggi, 2005) and/or whether the observed efficiency is related to intelligence or other, more personality-related factors. These findings not only shed light on the regions mediating processing at capacity limits, but also underline the importance of the interindividualdifferences approach in neuroimaging research.

\section{AUTHOR NOTE}

This study was supported by the Swiss National Science Foundation (Grant 101211-101849) and the Bern University Research Foundation. The authors thank their participants for their time and effort; the neuroradiology team for their technical assistance; Katharina Henke and John Jonides, as well as two anonymous reviewers, for thoughtful comments on an earlier draft; and Mirjam Kurth for language-related advice. Correspondence concerning this article should be addressed to S. M. Jaeggi, Department of Psychology, Division of Experimental Psychology and Neuropsychology, University of Bern, Muesmattstrasse 45, CH-3012 Bern, Switzerland (email: susanne.jaeggi@psy.unibe.ch).

\section{REFERENCES}

Bor, D., Cumming, N., Scott, C. E. L., \& Owen, A. M. (2004). Prefrontal cortical involvement in verbal encoding strategies. European Journal of Neuroscience, 19, 3365-3370.

Bor, D., \& Owen, A. M. (2007). A common prefrontal-parietal network for mnemonic and mathematical recoding strategies within working memory. Cerebral Cortex, 17, 778-786.

Bosshardt, S., Degonda, N., Schmidt, C. F., Boesiger, P., Nitsch, R. M., Hock, C., \& Henke, K. (2005). One month of human memory consolidation enhances retrieval-related hippocampal activity. Hippocampus, 15, 1026-1040.

Braver, T. S., Cohen, J. D., Nystrom, L. E., Jonides, J., Smith, E. E., \& NolL, D. C. (1997). A parametric study of prefrontal cortex involvement in human working memory. NeuroImage, 5, 49-62.

CABEZA, R. (2002). Hemispheric asymmetry reduction in older adults: The HAROLD model. Psychology \& Aging, 17, 85-100.

Cabeza, R., Grady, C. L., Nyberg, L., McIntosh, A. R., Tulving, E., KAPUR, S., ET AL. (1997). Age-related differences in neural activity during memory encoding and retrieval: A positron emission tomography study. Journal of Neuroscience, 17, 391-400.

Cabeza, R., \& NyberG, L. (2000). Imaging cognition II: An empirical review of 275 PET and fMRI studies. Journal of Cognitive Neuroscience, 12, 1-47.

Callicott, J. H., Bertolino, A., Mattay, V. S., Langheim, F. J., DuYn, J., Coppola, R., ET AL. (2000). Physiological dysfunction of the dorsolateral prefrontal cortex in schizophrenia revisited. Cerebral Cortex, 10, 1078-1092.

Callicott, J. H., Mattay, V. S., Bertolino, A., Finn, K., Coppola, R., FRANK, J. A., ET AL. (1999). Physiological characteristics of capacity constraints in working memory as revealed by functional MRI. Cerebral Cortex, 9, 20-26.

Carlson, S., Martinkauppi, S., Rama, P., Salli, E., Korvenoja, A., \& Aronen, H. J. (1998). Distribution of cortical activation during visuospatial $n$-back tasks as revealed by functional magnetic resonance imaging. Cerebral Cortex, 8, 743-752.

Caviness, V. S., JR., Meyer, J., Makris, N., \& Kennedy, D. N. (1996). MRI-based topographic parcellation of human neocortex: An anatomically specified method with estimate of reliability. Journal of Cognitive Neuroscience, 8, 566-587.

Cowan, N. (2001). The magical number 4 in short-term memory: A reconsideration of mental storage capacity. Behavioral \& Brain Sciences, 24, 87-185.

Cowan, N. (2005). Working memory capacity. New York: Psychology Press.

Cowan, N., Elliott, E. M., Scott Saults, J., Morey, C. C., Mattox, S.,
Hismuatullina, A., \& Conway, A. R. (2005). On the capacity of attention: Its estimation and its role in working memory and cognitive aptitudes. Cognitive Psychology, 51, 42-100.

Daneman, M., \& Carpenter, P. A. (1980). Individual differences in working memory and reading. Journal of Verbal Learning \& Verbal Behavior, 19, 450-466.

D'Esposito, M. (2001). Working memory. In R. Cabeza \& A. Kingstone (Eds.), Handbook of functional neuroimaging of cognition (pp. 293327). Cambridge, MA: MIT Press.

Dobbins, I. G., Rice, H. J., Wagner, A. D., \& Schacter, D. L. (2003). Memory orientation and success: Separable neurocognitive components underlying episodic recognition. Neuropsychologia, 41, 318333.

Duncan, J., \& Owen, A. M. (2000). Common regions of the human frontal lobe recruited by diverse cognitive demands. Trends in Neurosciences, 23, 475-483.

EgNER, T., \& Hirsch, J. (2005). Where memory meets attention: Neural substrates of negative priming. Journal of Cognitive Neuroscience, 17, 1774-1784.

Frith, C., \& Dolan, R. (1996). The role of the prefrontal cortex in higher cognitive functions. Cognitive Brain Research, 5, 175-181.

Garavan, H., Ross, T. J., Murphy, K., Roche, R. A. P., \& Stein, E. A. (2002). Dissociable executive functions in the dynamic control of behavior: Inhibition, error detection, and correction. NeuroImage, 17, 1820-1829.

Goldberg, T. E., Berman, K. F., Fleming, K., Ostrem, J., Van Horn, J. D., Esposito, G., ET AL. (1998). Uncoupling cognitive workload and prefrontal cortical physiology: A PET rCBF study. NeuroImage, 7, 296-303.

Haier, R. J., Siegel, B. V., Jr., Maclachlan, A., Soderling, E., Lottenberg, S., \& Buchsbaum, M. S. (1992). Regional glucose metabolic changes after learning a complex visuospatial/motor task: A positron emission tomographic study. Brain Research, 570, 134143.

INGVAR, D. H. (1994). The will of the brain: Cerebral correlates of willful acts. Journal of Theoretical Biology, 171, 7-12.

JAEGGI, S. M. (2005). Capacity limitations in human cognition: Behavioural and biological contributions. Unpublished doctoral dissertation, University of Bern.

Jaeggi, S. M., Seewer, R., Nirkko, A. C., Eckstein, D., Schroth, G., Groner, R., \& Gutbrod, K. (2003). Does excessive memory load attenuate activation in the prefrontal cortex? Load-dependent processing in single and dual tasks: Functional magnetic resonance imaging study. NeuroImage, 19, 210-225.

Jansma, J. M., Ramsey, N. F., Slagter, H. A., \& Kahn, R. S. (2001). Functional anatomical correlates of controlled and automatic processing. Journal of Cognitive Neuroscience, 13, 730-743.

Jansma, J. M., Ramsey, N. F., van der Wee, N. J. A., \& Kahn, R. S. (2004). Working memory capacity in schizophrenia: A parametric fMRI study. Schizophrenia Research, 68, 159-171.

Johnson, M. K., Hashtroudi, S., \& Lindsay, D. S. (1993). Source monitoring. Psychological Bulletin, 114, 3-28.

Jonides, J., \& NeE, D. E. (2006). Brain mechanisms of proactive interference in working memory. Neuroscience, 139, 181-193.

Jonides, J., Schumacher, E. H., Smith, E. E., Lauber, E. J., Awh, E., Minoshima, S., \& Koeppe, R. A. (1997). Verbal working memory load affects regional brain activation as measured by PET. Journal of Cognitive Neuroscience, 9, 462-475.

Kane, M. J., \& Engle, R. W. (2002). The role of prefrontal cortex in working-memory capacity, executive attention, and general fluid intelligence: An individual-differences perspective. Psychonomic Bulletin \& Review, 9, 637-671.

Kassubek, J., Schmidtke, K., Kimmig, H., Lucking, C. H., \& GreenleE, M. W. (2001). Changes in cortical activation during mirror reading before and after training: An fMRI study of procedural learning. Cognitive Brain Research, 10, 207-217.

KIRCHNER, W. K. (1958). Age differences in short-term retention of rapidly changing information. Journal of Experimental Psychology, 55, 352-358.

Kyllonen, P. C., \& Christal, R. E. (1990). Reasoning ability is (little more than) working-memory capacity? Intelligence, 14, 389-433.

Li, S. C., \& Lindenberger, U. (1999). Cross-level unification: A com- 
putational exploration of the link between deterioration of neurotransmitter systems and dedifferentiation of cognitive abilities in old age. In L. G. Nilsson \& H. J. Markowitsch (Eds.), Cognitive neuroscience of memory (pp. 103-146). Seattle: Hogrefe \& Huber.

Linden, D. E., Bittner, R. A., Muckli, L., Waltz, J. A., Krieges-Korte, N., Goebel, R., ET AL. (2003). Cortical capacity constraints for visual working memory: Dissociation of fMRI load effects in a fronto-parietal network. NeuroImage, 20, 1518-1530.

Mitchell, K. J., Johnson, M. K., Raye, C. L., \& Greene, E. J. (2004). Prefrontal cortex activity associated with source monitoring in a working memory task. Journal of Cognitive Neuroscience, 16, 921-934.

Nebel, K., Wiese, H., Stude, P., de Greiff, A., Diener, H. C., \& KeIDEL, M. (2005). On the neural basis of focused and divided attention. Cognitive Brain Research, 25, 760-776.

Neubauer, A. C., Grabner, R. H., Fink, A., \& Neuper, C. (2005). Intelligence and neural efficiency: Further evidence of the influence of task content and sex on the brain-IQ relationship. Cognitive Brain Research, 25, 217-225.

NiRKKo, A. C. (2000). A small software utility for fully automated download and evaluation of fMRI data. NeuroImage, 11, S919.

NirkKo, A. C., Ozdoba, C., Redmond, S. M., Burki, M., Schroth, G., Hess, C. W., \& Wiesendanger, M. (2001). Different ipsilateral representations for distal and proximal movements in the sensorimotor cortex: Activation and deactivation patterns. NeuroImage, 13, 825835 .

Nystrom, L. E., Braver, T. S., Sabb, F. W., Delgado, M. R., Noll, D. C., \& Cohen, J. D. (2000). Working memory for letters, shapes, and locations: fMRI evidence against stimulus-based regional organization in human prefrontal cortex. NeuroImage, 11, 424-446.

OldFIELD, R. C. (1971). The assessment and analysis of handedness: The Edinburgh Inventory. Neuropsychologia, 9, 97-113.

Owen, A. M., McMillan, K. M., Laird, A. R., \& Bullmore, E. (2005). $\mathrm{N}$-back working memory paradigm: A meta-analysis of normative functional neuroimaging studies. Human Brain Mapping, 25, 46-59.

Perlstein, W. M., Carter, C. S., Noll, D. C., \& Cohen, J. D. (2001). Relation of prefrontal cortex dysfunction to working memory and symptoms in schizophrenia. American Journal of Psychiatry, 158, 1105-1113.

Prince, S. E., Daselaar, S. M., \& Cabeza, R. (2005). Neural correlates of relational memory: Successful encoding and retrieval of semantic and perceptual associations. Journal of Neuroscience, 25, 1203-1210.

Qin, Y., Carter, C. S., Silk, E. M., Stenger, V. A., Fissell, K., Goode, A., \& ANDERson, J. R. (2004). The change of the brain activation patterns as children learn algebra equation solving. Proceedings of the National Academy of Sciences, 101, 5686-5691.

Rademacher, J., Galaburda, A. M., Kennedy, D. N., Filipek, P. A., \& CAVInESS, V. S., JR. (1992). Human cerebral cortex: Localization, parcellation, and morphometry with magnetic resonance imaging. Journal of Cognitive Neuroscience, 4, 352-374.
Ranganath, C., Johnson, M. K., \& D'Esposito, M. (2000). Left anterior prefrontal activation increases with demands to recall specific perceptual information. Journal of Neuroscience, 20, RC108.

Rubia, K., Smith, A. B., Brammer, M. J., \& Taylor, E. (2003). Right inferior prefrontal cortex mediates response inhibition while mesial prefrontal cortex is responsible for error detection. NeuroImage, 20, 351-358.

Rypma, B., Berger, J. S., \& D'Esposito, M. (2002). The influence of working-memory demand and subject performance on prefrontal cortical activity. Journal of Cognitive Neuroscience, 14, 721-731.

Rypma, B., \& D'Esposito, M. (1999). The roles of prefrontal brain regions in components of working memory: Effects of memory load and individual differences. Proceedings of the National Academy of Sciences, 96, 6558-6563.

Rypma, B., \& D'Esposito, M. (2000). Isolating the neural mechanisms of age-related changes in human working memory. Nature Neuroscience, 3, 509-515.

Schacter, D. L., \& Buckner, R. L. (1998). Priming and the brain. Neuron, 20, 185-195.

Schumacher, E. H., Lauber, E., Awh, E., Jonides, J., Smith, E. E., \& KoEPPE, R. A. (1996). PET evidence for an amodal verbal working memory system. NeuroImage, 3, 79-88.

Smith, E. E., Geva, A., Jonides, J., Miller, A., Reuter-Lorenz, P., \& KoEpre, R. A. (2001). The neural basis of task-switching in working memory: Effects of performance and aging. Proceedings of the National Academy of Sciences, 98, 2095-2100.

Snodgrass, J. G., \& Corwin, J. (1988). Pragmatics of measuring recognition memory: Applications to dementia and amnesia. Journal of Experimental Psychology: General, 117, 34-50.

TodD, J. J., \& Marois, R. (2004). Capacity limit of visual short-term memory in human posterior parietal cortex. Nature, 428, 751-754.

van der Graaf, F. H. C. E., De Jong, B. M., Maguire, R. P., Meiners, L. C., \& LEENDERS, K. L. (2004). Cerebral activation related to skills practice in a double serial reaction time task: Striatal involvement in random-order sequence learning. Cognitive Brain Research, 20, 120-131.

Vogel, E. K., McCollough, A. W., \& Machizawa, M. G. (2005). Neural measures reveal individual differences in controlling access to working memory. Nature, 438, 500-503.

WAGER, T. D., \& SMITH, E. E. (2003). Neuroimaging studies of working memory: A meta-analysis. Cognitive, Affective, \& Behavioral Neuroscience, 3, 255-274.

Wood, J. N., \& Grafman, J. (2003). Human prefrontal cortex: Processing and representational perspectives. Nature Reviews Neuroscience, 4, 139-147.

Xu, Y., \& Chun, M. M. (2006). Dissociable neural mechanisms supporting visual short-term memory for objects. Nature, 440, 91-95.

(Manuscript received February 2, 2006; revision accepted for publication September 21, 2006.) 